\title{
Callose Synthase Family Genes Involved in the Grapevine Defense Response to Downy Mildew Disease
}

\author{
Ying Yu, Li Jiao, Shufang Fu, Ling Yin, Yali Zhang, and Jiang Lu
}

First, second, third, fourth, fifth, and sixth authors: The Viticulture and Enology Program, College of Food Science and Nutritional Engineering, China Agricultural University, Beijing, 100083, China; first author: Institute of Genetics and Developmental Biology, China Academy of Science; and fourth author: Guangxi Crop Genetic Improvement and Biotechnology Key Lab, Guangxi Academy of Agricultural Sciences. Accepted for publication 12 October 2015.

\begin{abstract}
Yu, Y., Jiao, L., Fu, S., Yin, L., Zhang, Y., and Lu, J. 2016. Callose synthase family genes involved in the grapevine defense response to downy mildew disease. Phytopathology 106:56-64.

The deposition of callose is a common plant defense response to intruding pathogens and part of the plant's innate immunity. In this study, eight grapevine callose synthase (CalS) genes were identified and characterized. To investigate biological function of CalS in grapevine against the infection of Plasmopara viticola, expression patterns of grapevine CalS family genes were analyzed among resistant/susceptible cultivars. After P. viticola infection, expression of CalS1, 3, 7, 8, 9, 10, and 11 were significantly modified among the grapevine cultivars. For

example, the expression of CalS1 and CalS10 were greatly increased in downy mildew (DM)-immune Muscadinia rotundifolia 'Carlos' and 'Noble'. Transient expression assay with promoters of the CalS1 and CalS10 genes confirmed that they were regulated by the oomycete pathogen $P$. viticola. CalS1 promoter activity was also significantly upregulated by $\mathrm{ABA}$ in DM-immune $M$. rotundifolia 'Noble', but downregulated in DM-susceptible Vitis vinifera 'Chardonnay'. The CalS1 promoter, however, was also down-regulated by GA in 'Chardonnay', but not affected in 'Noble'. The promoter activity of CalS10 was significantly up-regulated by GA in 'Chardonnay', but not regulated by ABA at all. It is proposed that CalS1 and CalS10 were involved in grapevine defense against DM disease.
\end{abstract}

Callose, a $\beta$-1,3-glucan, exists in most plant tissues and plays an important role in plant defense response (Chen and Kim 2009; Eshraghi et al. 2014; Li et al. 2010). It has been reported that callose could be deposited between the plasma membrane and cell wall or around the stomata after pathogen attack (Brown et al. 1998; Gindro et al. 2003; Gomez-Gomez et al. 1999). Its deposition could impede the microbial penetration as a physical barrier or inhibit the development of pathogen haustoria by preventing it from obtaining nutrients of the host cells (Donofrio and Delaney 2001; Yang et al. 2014). The deposition of callose could be regulated by conserved pathogen-associated molecular patterns, hormone signals such as ABA, resistance-associated chemicals and secondary metabolites such as the nonprotein amino acid $\beta$-amino butyric acid (BABA) and the 4-methoxylated indole glucosinolates (Clay et al. 2009; Flors et al. 2008; Ton and Mauch-Mani 2004; Ton et al. 2009).

Callose is synthesized by callose synthases (CalS), which contains multiple transmembrane segments and a hydrophilic central loop (Kelly et al. 1996; Verma and Hong 2001). In Arabidopsis thaliana, 12 callose synthase genes were identified and characterized (Richmond and Somerville 2000). Among them, the biological role of AtCalS12/GSL5 (first described as PMR4; POWDERY MILDEW RESISTANT4) was well studied in stress and pathogen response. This gene is required for wound and papillary callose formation in response to fungal pathogens and transported from the plasma membrane to the site of attempted

Corresponding author: J. Lu; E-mail address: jiangluvitis@cau.edu.cn

First and second authors contributed equally to this work.

*The $\boldsymbol{e}$-Xtra logo stands for "electronic extra" and indicates that three supplementary figures and three supplementary tables are published online.

http://dx.doi.org/10.1094/PHYTO-07-15-0166-R

(c) 2016 The American Phytopathological Society fungal penetration during powdery mildew infection (Ellinger et al. 2013; Luna et al. 2011; Nishimura et al. 2003). AtCalS12/GSL5 gene could also be up-regulated by salicylic acid (SA) treatment and this induction is Npr1 (nonexpresser of PR genes) dependent (Dong et al. 2008). In addition, AtCalS 1, 5, 9, and 10 (GSL 6, 2, 10, and 8 ) were induced by the oomycete pathogen Peronospora parasitica that causes downy mildew (DM) disease of $A$. thaliana. Their expression levels were also affected by SA treatment and the most significant induction was observed in AtCalS1/GSL6. Promoter activity analysis indicated that the high induction of AtCalS1/GSL6 by SA treatment requires Npr1 (Jacobs et al. 2003). In other plants, silencing of ClCalS1 in Citrus limon increased plant susceptibility to Xanthomonas citri subsp. citri, suggesting that the plant cell wall associated defense is the principal initial barrier against Xanthomonas infection in citrus plants (Enrique et al. 2011). Similarly, in wheat spike, the suppression of callose synthase activity by free fatty acids could alter the resistance of wheat to $F$. graminearum (Blümke et al. 2014). In addition to the defense response, callose synthase genes were also reported to mediate the synthesis of callose during plant development. For example, AtCalS5/GSL2 played an important role in the synthesis of exine of pollen wall and the viability of the pollen (Dong et al. 2005). OsGSL5 from rice and NaGSL1 from tobacco, orthologs of the AtCalS5/GSL2 gene, were also highly expressed in developing anthers (Brownfield et al. 2007; Doblin et al. 2001; Yamaguchi et al. 2006).

To elucidate the molecular mechanism of functional genes in plants, transgenic studies are found as an efficient system, including model plant species Arabidopsis and tobacco (Liu et al. 2005; Malnoy et al. 2003), and crop species pepper and rice (Lee and Hwang 2006; Li et al. 2005). Compared with the stable genetic transformation, transient expression is a time saving approach. For instance, transient expression of $V v N P R l$ in grapevine leaves could enhance plant resistance to fungal infection (Henanff et al. 2009). Transient expression of a glyoxal 
oxidase gene isolated from a disease resistant grape species Vitis pseudoreticulata in a susceptible genotype was found to enhance defense against powdery mildew (Guan et al. 2011). Similarly, the transient expression technology was used to study promoter activities. For example, studying the promoter activity of a stilbene synthase gene from V. pseudoreticulata (VpSTS) using Agrobacteriummediated transient transformation system revealed that $V p S T S$ was induced by infection, salicylic acid, and low temperature (Xu et al. 2010).

DM, caused by Plasmopara viticola, is the most destructive grapevine disease in viticulture areas with warm and humid climate conditions (Boso and Kassemeyer 2008). A previous study demonstrated that callose was mainly deposited in the leaf surface near stomata among DM-resistant grapevines, but less so in the DM-susceptible grapevines after $P$. viticola incubation (Yu et al. 2012). To further understand the mechanism of callose deposition in grapevine against DM disease, this study was designed to investigate the expression and regulation of grapevine callose synthase family genes in response to $P$. viticola pathogen infection and disease development.

\section{MATERIALS AND METHODS}

Plant materials and plant treatments. Two DM-immune M. rotundifolia cultivars ('Noble' and 'Carlos'), the DM-resistant and the partially resistant V. amurensis 'Shuanghong' and 'Zuoshanyi', and a DM-susceptible $V$. vinifera 'Chardonnay' were maintained in 1 gallon pots in glasshouse at China Agricultural University, Beijing. The potted grapevines were transferred to a big plant growth chamber $\left(16 \mathrm{~h}\right.$ light, $28^{\circ} \mathrm{C} / 8 \mathrm{~h}$ dark, $25^{\circ} \mathrm{C}$ ) for $P$. viticola inoculation and incubation.

$P$. viticola inoculum was collected from an experimental vineyard in Beijing. The third to fifth unfolded leaves from the apex were sprayed with a $P$. viticola suspension of $1 \times 10^{5}$ sporangia $/ \mathrm{ml}$. The inoculated leaves were covered with plastic bags to maintain high humidity. Control plants were inoculated with sterile distilled water and maintained under the same conditions. Samples were collected at 6 and $12 \mathrm{~h}$ postinoculation (hpi), 1, 3, 5, and 8 days postinoculation (dpi), and were immediately frozen in liquid nitrogen and stored at $-80^{\circ} \mathrm{C}$ until utilization. All experiments were repeated three times.

For ABA treatment, the third to fifth unfolded leaves from the shoot apex were sprayed with $100 \mu \mathrm{M} \mathrm{ABA}$ and were harvested $12 \mathrm{~h}$ after treatment. Leaves sprayed with water at the same time point were used as control. The experiment was repeated three times.

DNA, RNA extraction, and real-time RT-PCR. Genomic DNA and total RNA were extracted from leaf tissues using the CTAB method (Murray and Thompson 1980). The first strand of cDNA was synthesized using M-MLV Reverse transcription (Promega). A grape elongation factor $(E F 1-\alpha)$ gene was used as an internal control for normalization (Jiao et al. 2015). The expression levels of the CalS genes in different grape cultivars were analyzed by real-time reverse transcription polymerase chain reaction (RT-PCR) using the specific primers shown in Supplementary Table S1. The specificity of each primer pair was evaluated through melting curve analysis and a single DNA fragment was amplified for each gene as only one melting peak was detected for each primer pair (Supplementary Fig. S1). For the pathogen treatment assay, data were quantified using $2^{-\Delta \Delta C T}$ method (Livak and Schmittgen 2001) and the level of the CalS transcript at $0 \mathrm{hpi}$ was set at 1.0. The relative expression was represented as the fold changes by comparing $P$. viticola-treated sample and water-treated sample at the same time point. All the statistical analysis for the data were performed with one-way analysis of variance (ANOVA) or Student's $t$ test using SPSS 16.0 (SPSS Inc.) at the 95\% confidence level.

Identification of CalS sequences. The CalS family genes of grape were identified using BlastN queries of grape genome (http://
www.genoscope.cns.fr/spip/Vitis-vinifera-whole-genome.html). The sequences of different CalS family genes from Arabidopsis, cotton, and barley were obtained from the NCBI database (http:// www.ncbi.nlm.nih.gov/). The multiple sequence alignment and phylogenetic tree construction were performed using ClustalX1.8 (Thompson et al. 1994) and MEGA4 (Tamura et al. 2007). The conserved domains within CalS proteins were online predicted using NCBI's Conserved Domain Database and SMART (http:// smart.embl-heidelberg.de/).

Promoter-GUS constructs. Promoter regions of the two genes, CalS1 and CalS10, were amplified by PCR using genomic DNA of grapevine leaves. The primers for each $\mathrm{CalS}$ gene promoter were designed based on the sequences $2-\mathrm{kb}$ upstream from the translational start codons (Supplementary Table S2). PCR-amplified fragments were cloned into PGEM-T Easy vector, verified by DNA sequencing, and subcloned into binary expression vector $\mathrm{pBI} 121$ as a fusion with $\beta$-glucuronidase (GUS, as a reporter) coding region. The $c$ is-acting elements prediction within the promoter region was analyzed using PlantCARE (http://bioinformatics.psb.ugent.be/ webtools/plantcare/html/).

GUS activity assay. The expression constructs were electroporated into Agrobacterium tumefaciens strain GV3101 and transformed into the first unfolded leaf of in vitro-grown $V$. vinifera 'Thompson Seedless' plantlets by the vacuum infiltration method (Santos-Rosa et al. 2008; Xu et al. 2010). After the vacuum infiltration, leaves were dried on sterile filter paper and transferred to $0.8 \%$ agar plates, which were incubated at $25^{\circ} \mathrm{C}$ in dark for 1 day, and then with a 16:8 h light/dark cycle for another day. After the incubation, leaves were inoculated with $P$. viticola suspension $(1 \times$ $10^{5}$ sporangia/ml) or sprayed with $1 \mathrm{mM} \mathrm{ABA} / 1 \mathrm{mM}$ GA. The treated leaves were kept in the $0.8 \%$ agar plates $\left(16 \mathrm{~h}\right.$ light, $28^{\circ} \mathrm{C} / 8 \mathrm{~h}$ dark, $25^{\circ} \mathrm{C}$ ). Samples for histochemical staining and GUS quantification were collected $24 \mathrm{~h}$ after treatments.

Histochemical staining of GUS expression was performed as described by Jefferson (1987). After staining, the chlorophyll was completely removed from leaf tissues by successive washes with 75 to $100 \%$ ethanol. Finally, pictures for the treated leaf samples were taken with a SONY camera. The quantification of GUS activity was measured as described by Jefferson (1987) and expressed as nanomoles of 4-methylumbelliferone (4-MU) produced per minute per milligram of protein.

\section{RESULTS}

Isolation and characterization of $\mathrm{CalS}$ in grapevine. When callose synthase sequences of $A$. thaliana were used in BlastN queries against the grape genome sequences (http://www. genoscope.cns.fr/spip/Vitis-vinifera-whole-genome.html), eight putative callose synthase $(\mathrm{CalS})$ genes were identified and were named according to the CalS nomenclature system used in Verma and Hong (2001). These VvCalS were designed as VvCalS1, VvCalS3, VvCalS5, VvCalS7, VvCalS8, VvCalS9, VvCalS10, and $V v C a l S 11$ based on their sequence similarity to AtCalS (Supplementary Table S3). Except $V v C a l S 11$ for which the chromosome location was unknown, the $V v C a l S$ genes were located on chromosomes $4,6,12,13,17$, and 19 . They contained 20 to 45 exons and 30 to 48 introns and their open reading frames (ORF) ranged from 4,908 to $6,159 \mathrm{bp}$, encoding 1,632 to 2,053 amino-acid polypeptides (Table 1).

One $\beta$-1,3-glucan synthase domain and several transmembrane domains which were conserved among CalS families were identified in $V v C a l S$. The transmembrane domains were positioned in both the $\mathrm{C}$-terminal and the $\mathrm{N}$-terminal of the protein (Fig. 1A). The sequence similarity among the $V v C a l S$ genes varied from 30 to $80 \%$. For example, $\mathrm{VvCalS1}$ shares $79.5 \%$ identity with $V v C a l S 3$, while $V v C a l S 5$ only shares $33.3 \%$ identity with VvCalS11. Phylogenetic analysis among the grape (Vitis vinifera) VvCalS, Arabidopsis (Arabidopsis thaliana) AtCalS, barley 
(Hordeum vulgare) HvCalS, and cotton (Gossypium hirsutum) GhCalS suggests that these CalS families could be divided into four groups and $V v C a l S$ genes were distributed among them all (Fig. 1B).

Expression of CalS genes in response to $P$. viticola infection. The expression patterns of grapevine CalS genes in response to $P$. viticola infection were analyzed among grapevines with different resistance levels, including the DM-immune $M$. rotundifolia 'Noble' and 'Carlos', the resistant $V$. amurensis 'Shuanghong', the partially resistant $V$. amurensis 'Zuoshanyi', and the susceptible $V$. vinifera 'Chardonnay'. At early $P$. viticola infection, CalS1 was significantly up-regulated in the DM-immune M. rotundifolia 'Carlos' (6 and $12 \mathrm{hpi}$ ) and 'Noble' (12 hpi), as well as the partially resistant $V$. amurensis 'Zuoshanyi' (12 hpi), but no significant changes were detected in other grape cultivars. With the exception of M. rotundifolia 'Noble', the expression level of CalS3 was significantly increased in all the cultivars. CalS7 was remarkably up-regulated in $M$. rotundifolia 'Carlos' (6 and $12 \mathrm{hpi}$ ) and the resistant V. amurensis 'Shuanghong' (6 and $12 \mathrm{hpi}$ ). The expression of CalS10 was significantly increased in the DM-immune muscadine cultivars at 6 and $12 \mathrm{hpi}$. Its expression was also up-regulated in $V$. amurensis 'Zuoshanyi' (12 hpi) and V. vinifera 'Chardonnay' (6 hpi) (Fig. 2).

The expression of other CalS genes, CalS8, CalS9, and CalS11, was not changed significantly in muscadine grapes at both 6 and 12 hpi. CalS8 was only up-regulated in the susceptible $V$. vinifera 'Chardonnay' (6 and $12 \mathrm{hpi}$ ). CalS9 was significantly up-regulated in resistant $V$. amurensis 'Shuanghong' at 12 hpi. Similarly, the expression of CalS11 was increased in 'Shuanghong' (12 hpi) and 'Chardonnay' (6 hpi) (Fig. 2). The expression of CalS5 was very low in all grape cultivars and no significant changes were found.

When CalS gene expressions of the grapevines were further analyzed from 1 to 8 days after $P$. viticola infection, CalS1 was upregulated in the $M$. rotundifolia 'Carlos' and 'Noble' at 1 dpi. The expression of the former continued to increase at $3 \mathrm{dpi}$ and up to

TABLE 1. CalS genes in Vitis vinifera 'Pinot noir'

\begin{tabular}{llcccc}
\hline Gene & \multicolumn{1}{c}{ Accession number } & Number of introns & Number of exons & ORF (bp) & Chromosome location \\
\hline$V v$ CalS1 & GSVIVT01001361001 & 41 & 42 & 5,760 & chr13:23971224.0.23998913 \\
$V v$ CalS3 & GSVIVT01025362001 & 41 & 42 & 5,847 & chr6:1470318.0.1508936 \\
VvCalS5 & GSVIVT01026489001 & 43 & 44 & 5,877 & chr4:22776367.0.22794921 \\
$V v$ CalS7 & GSVIVT01020854001 & 43 & 44 & 5,694 & chr12:995099.0.1078999 \\
$V v$ CalS8 & GSVIVT01000622001 & 44 & 45 & 6,159 & chr19:13989275.0.14016964 \\
$V v$ CalS9 & GSVIVT01025370001 & 30 & 29 & 5,715 & chr6:1350308.0.1408792 \\
$V v$ CalS10 & GSVIVT01007560001 & 48 & 49 & 5,085 & chr17:12030876.0.12098518 \\
V CalS11 & GSVIVT01005204001 XM_002263721.1 & - & - & 4,908 & ChrUn \\
\hline
\end{tabular}

a The number to each $V v$ CalS gene was assigned on the basis of homology with AtCalS.

$\mathrm{b}$ The open reading frame (ORF) of $V v$ CalS11 was obtained by splicing.
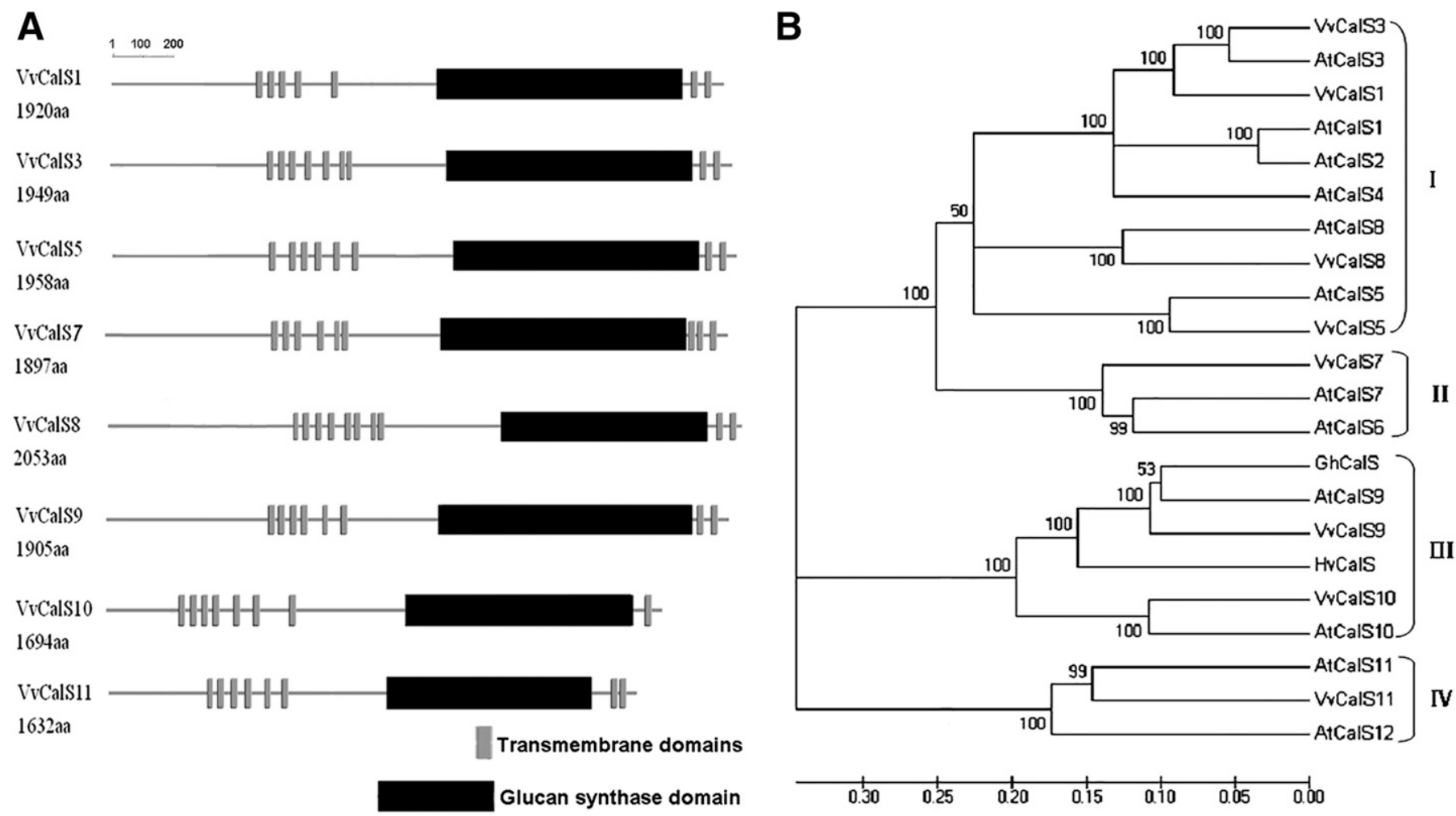

Fig. 1. Sequence analysis of grapevine callose synthase. A, Structure domain of VvCalS proteins. The deduced amino acid sequences of Vitis CalS genes were analyzed by using the SMART search. Transmembrane domains were indicated by the vertical gray bars and glucan synthase domains were indicated by the long black rectangle. B, Phylogenetic analysis of grapevine, Arabidopsis, barley, and cotton CalS proteins using MEGA neighbor-joining. Numbers at the branch points are bootstrap values. VvCalS: Vitis vinifera CalS protein; AtCalS: Arabidopsis thaliana CalS proteins, AtCalS1 (AF237733.1), AtCalS2 (NM_179847.1), AtCalS3 (NM_121303.6), AtCalS4 (NM_123045.2), AtCalS5 (NM_179622.2), AtCalS6 (NM_115772.2), AtCalS7 (NM_100528.1), AtCalS8 (NM_112317.1), AtCalS9 (NM_111596.5), AtCalS10 (GQ373182.1), AtCalS11 (NM_116736.1), and AtCalS12 (NM_116593.3); HvCalS: Hordeum vulgare CalS protein (AY177665); and GhCalS: Gossypium hirsutum CalS protein (AF085717). 
sixfold at 5 and $8 \mathrm{dpi}$, while the later was returned to normal expression level after 1 dpi. The expression of CalS1 was not changed significantly in other cultivars. CalS7 was significantly upregulated at 1,3 , and $5 \mathrm{dpi}$, but down-regulated at $8 \mathrm{dpi}$ in the M. rotundifolia 'Carlos'. Interestingly, it was down-regulated in another muscadine grape 'Noble' at all the sampling time points. In the partially resistant $V$. amurensis 'Zuoshanyi', the expression of CalS7 was increased at $1 \mathrm{dpi}$ and then decreased at $3 \mathrm{dpi}$. The expression of CalS10 was dramatically increased in the DMimmune $M$. rotundifolia 'Carlos' at 1, 3, and 5 dpi and 'Noble' at 3, 5 , and 8 dpi. In the DM-resistant $V$. amurensis 'Shuanghong', its expression was significantly up-regulated at $8 \mathrm{dpi}$. In the partially resistant $V$. amurensis 'Zuoshanyi', CalS10 was up-regulated at 1 and 8 dpi. No changes of CalS10 were found in the DM-susceptible Chardonnay (Fig. 3).

The other three grape CalS genes, CalS3, CalS8, and CalS11, were not regulated significantly by $P$. viticola in two immune muscadine grapes. CalS3 was up-regulated in $V$. amurensis 'Shuanghong' (1 dpi), 'Zuoshanyi' (1 and 8 dpi) and V. vinifera 'Chardonnay' ( 1 and 3 dpi). The expression of CalS8 was increased in 'Zuoshanyi' (1, 5, and $8 \mathrm{dpi}$ ) and V. vinifera 'Chardonnay' ( 3 and $5 \mathrm{dpi}$ ), but decreased in V. amurensis 'Shuanghong' $(1,5$, and $8 \mathrm{dpi})$. CalS11 was up-regulated in 'Shuanghong' (1 dpi), but downregulated in 'Zuoshanyi' (3 dpi). However, the expression of CalS9 increased at 3 and 5 dpi in the immune $M$. rotundifolia 'Noble', but decreased at 3 dpi in the partially resistant $V$. amurensis 'Zuoshanyi'
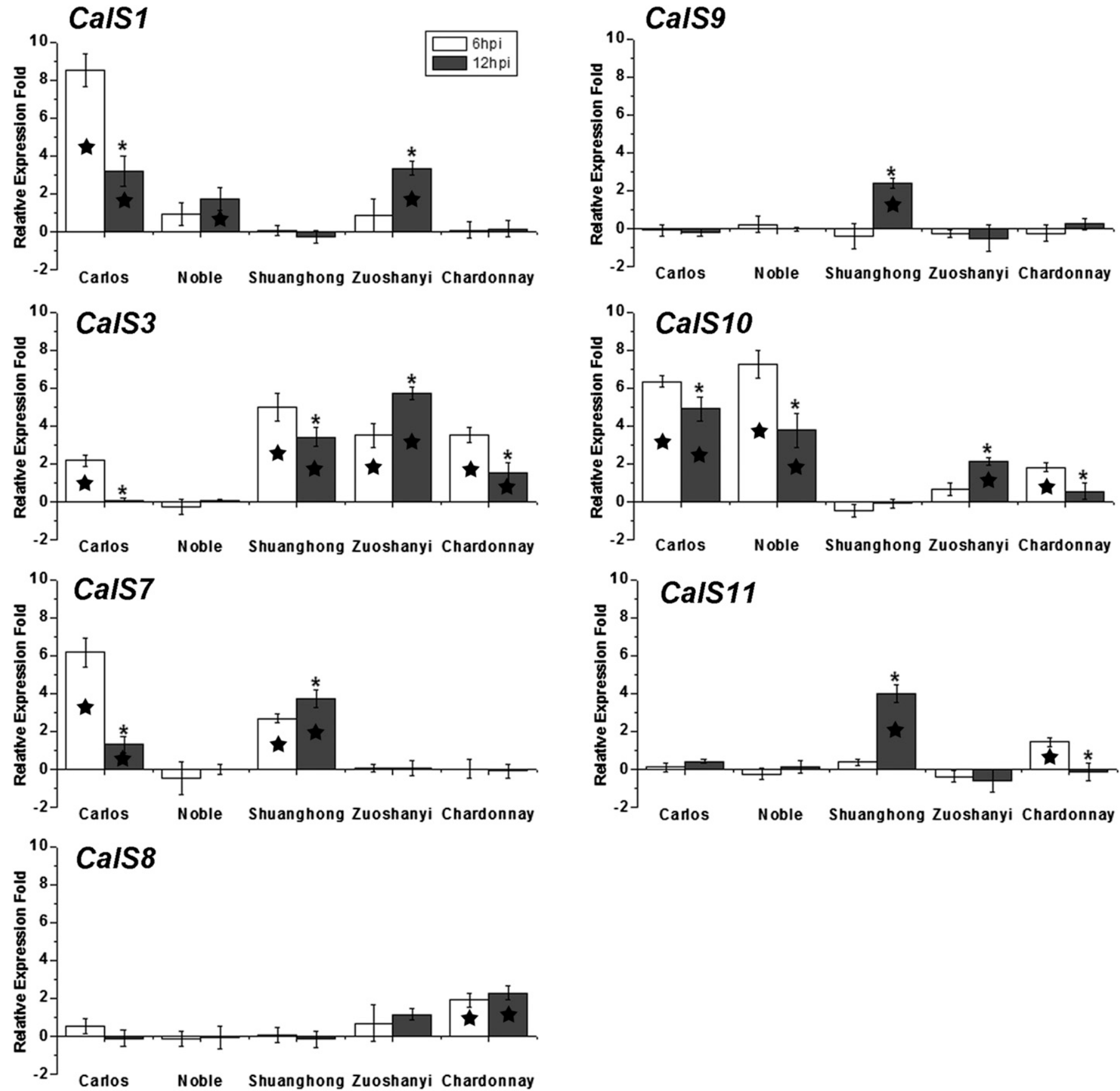

Fig. 2. Expression analysis of CalS genes among resistant/susceptible cultivars during the early infection. Grapevine leaves were collected at 6 and 12 h postPlasmopara viticola inoculation. The mRNA levels were determined by quantitative real-time polymerase chain reaction analysis. The relative expression was represented as the fold changes by comparing $P$. viticola-treated sample and water-treated sample at the same time point. $\star$ indicates a significant difference of gene expression between the $P$. viticola-treated sample and the water-treated sample at the same time point $(P=0.05)$. * indicates a significant difference between $6 \mathrm{~h}$ postinoculation (hpi) and $12 \mathrm{hpi}$ at the 0.05 level. 
(Fig. 3). CalS5 was expressed in a very low level in these grape cultivars at all sampling time points.

Isolation and sequence analysis of CalS1 and CalS10 gene promoter. Promoters of CalS1 and CalS10 genes were chosen for investigating the regulation of gene expression patterns associated with $P$. viticola infection. Sequences of 2-kb upstream from the translational start codons of $V v C a l S 1$ and $V v C a l S 10$ were amplified from the same five grape cultivars as above. As expected, promoter sequences within the same species are more similar than those amplified from different species (Supplementary Figs. S2 and
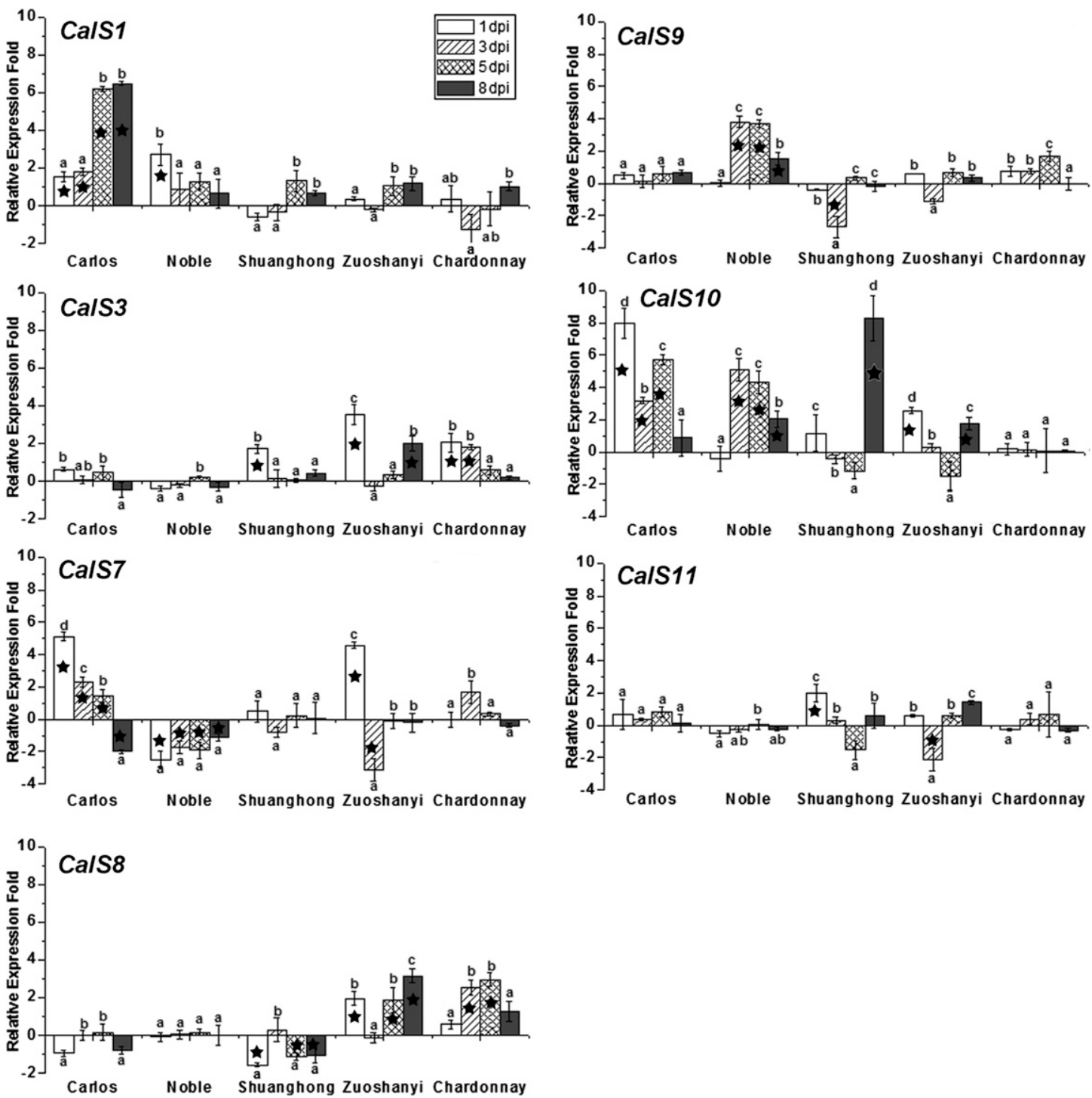

Fig. 3. Expression analysis of CalS genes among resistant/susceptible cultivars. Grapevine leaves were collected at 1, 3, 5, and 8 days post-Plasmopara viticola inoculation. The mRNA levels were determined by quantitative real-time polymerase chain reaction analysis. The relative expression was represented as the fold changes by comparing $P$. viticola-treated sample and water-treated sample at the same time point. $\star$ indicates a significant difference of gene expression between the $P$. viticola-treated sample and the water-treated sample at the same time point $(P=0.05)$. Different letters in each cultivar are significantly different among different time points at the 0.05 level according to analysis of variance.

TABLE 2. Cis-elements associated with pathogen- and stress-responsiveness in CalS1 promoter among five cultivars

\begin{tabular}{|c|c|c|c|c|c|c|}
\hline \multirow[b]{2}{*}{ Cis-element } & \multicolumn{5}{|c|}{ Number of cis-elements } & \multirow[b]{2}{*}{ Characterization } \\
\hline & Noble & Carlos & Shuanghong & Zuoshanyi & Chardonnay & \\
\hline ABRE & 1 & 1 & 0 & 0 & 0 & Cis-acting element involved in the abscisic acid responsiveness \\
\hline ARE & 1 & 1 & 2 & 2 & 0 & Cis-acting regulatory element essential for the anaerobic induction \\
\hline CGTCA-motif & 1 & 1 & 1 & 1 & 1 & Cis-acting regulatory element involved in the MeJA-responsiveness \\
\hline GARE-motif & 2 & 2 & 4 & 4 & 4 & Gibberellin-responsive element \\
\hline HSE & 0 & 0 & 0 & 1 & 1 & Cis-acting element involved in heat stress responsiveness \\
\hline LTR & 0 & 0 & 1 & 1 & 0 & Cis-acting element involved in low-temperature responsiveness \\
\hline MBS & 0 & 0 & 2 & 2 & 2 & MYB binding site involved in drought-inducibility \\
\hline P-box & 2 & 2 & 2 & 2 & 2 & Gibberellin-responsive element \\
\hline TC-rich repeats & 2 & 2 & 2 & 2 & 2 & Cis-acting element involved in defense and stress responsiveness \\
\hline TCA-element & 3 & 3 & 3 & 4 & 4 & Cis-acting element involved in salicylic acid responsiveness \\
\hline TGA-element & 1 & 1 & 1 & 1 & 1 & Auxin-responsive element \\
\hline TGACG-motif & 1 & 1 & 1 & 1 & 1 & Cis-acting regulatory element involved in the MeJA-responsiveness \\
\hline WUN-motif & 1 & 1 & 1 & 2 & 1 & Wound-responsive element \\
\hline
\end{tabular}


S3). When the CalS1 promoter sequences of muscadine grapes were compared with those in other cultivars, several large fragment deletions, several small fragment insertions, and more than 50 SNPs were identified. The $c i s$-acting elements analysis demonstrated that several stress- and hormone-regulated elements, such as the abscisic acid-responsive element ABRE, the MeJA-responsive element CGTCA-motif, the gibberellin-responsive element GARE-motif and the salicylic acid responsive element TCA, were detected in the CalS1 promoters. While the cis-elements were identified among all five cultivars studied, ABRE could only be found in DM-immune

TABLE 3. Cis-elements associated with pathogen- and stress-responsiveness in CalS10 promoter among five cultivars

\begin{tabular}{|c|c|c|c|c|c|c|}
\hline \multirow[b]{2}{*}{ Cis-element } & \multicolumn{5}{|c|}{ Number of cis-elements } & \multirow[b]{2}{*}{ Characterization } \\
\hline & Noble & Carlos & Shuanghong & Zuoshanyi & Chardonnay & \\
\hline ABRE & 1 & 1 & 1 & 3 & 1 & Cis-acting element involved in the abscisic acid responsiveness \\
\hline ARE & 3 & 4 & 3 & 2 & 3 & Cis-acting regulatory element essential for the anaerobic induction \\
\hline Box-W1 & 2 & 2 & 2 & 2 & 2 & Fungal elicitor responsive element \\
\hline CGTCA-motif & 2 & 2 & 2 & 2 & 2 & Cis-acting regulatory element involved in the MeJA-responsiveness \\
\hline ERE & 2 & 2 & 2 & 2 & 2 & Ethylene-responsive element \\
\hline GARE-motif & 0 & 0 & 1 & 1 & 1 & Gibberellin-responsive element \\
\hline HSE & 4 & 4 & 5 & 5 & 4 & Cis-acting element involved in heat stress responsiveness \\
\hline LTR & 2 & 1 & 1 & 1 & 1 & Cis-acting element involved in low-temperature responsiveness \\
\hline MBS & 3 & 3 & 3 & 3 & 3 & MYB binding site involved in drought-inducibility \\
\hline TC-rich repeats & 3 & 3 & 2 & 3 & 3 & Cis-acting element involved in defense and stress responsiveness \\
\hline TCA-element & 1 & 1 & 0 & 1 & 1 & Cis-acting element involved in salicylic acid responsiveness \\
\hline TGACG-motif & 2 & 2 & 2 & 2 & 2 & Cis-acting regulatory element involved in the MeJA-responsiveness \\
\hline
\end{tabular}

A
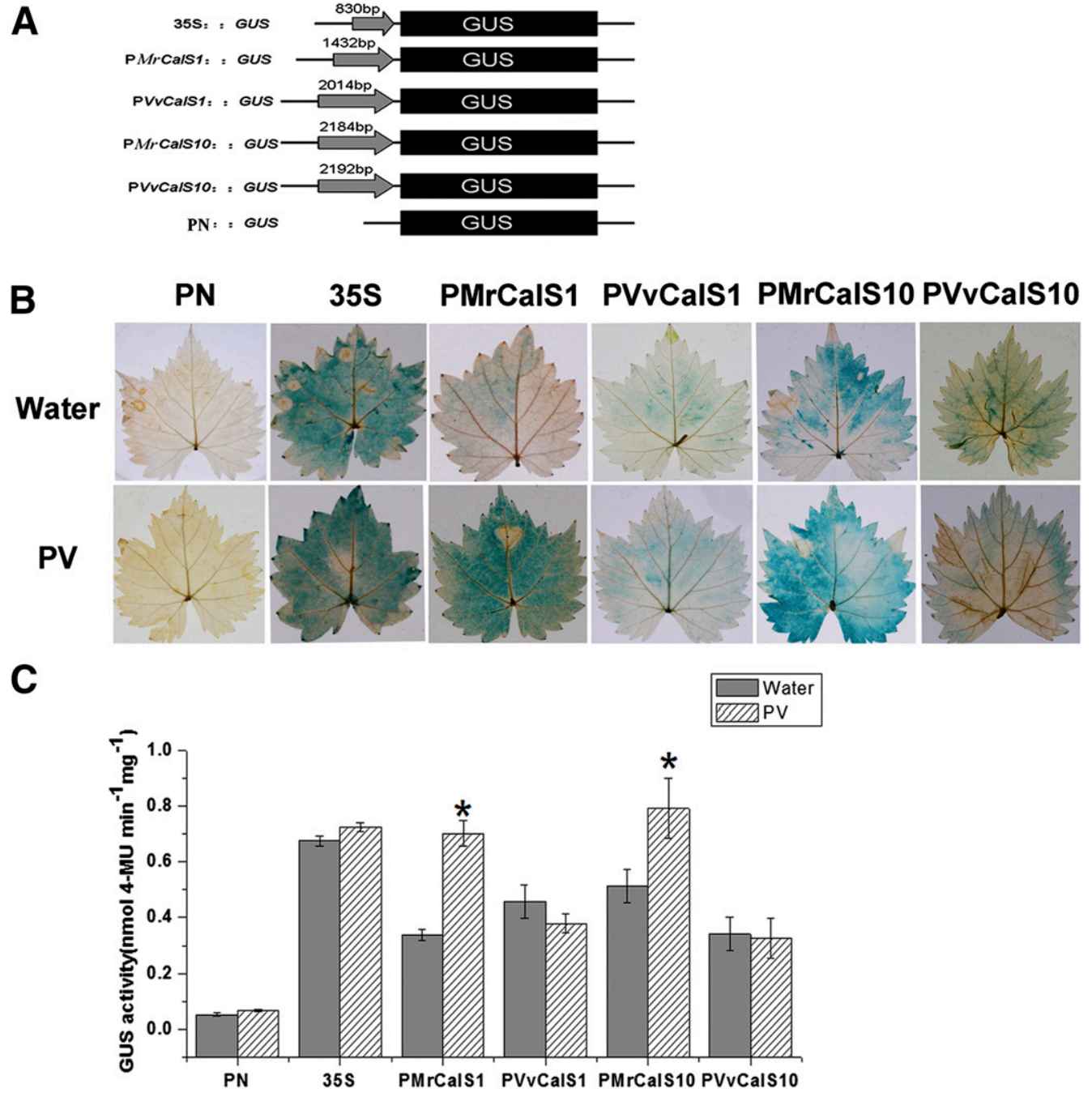

Fig. 4. GUS activity analysis after Plasmopara viticola infection. A, Structural diagram of vectors used in this experiment. 35S::GUS, vector pBI121; PMrCalS1, CalS1 promoter from Muscadinia rotundifolia 'Noble'; PVvCalS1, CalS1 promoter from Vitis vinifera 'Chardonnay'; PMrCalS10, CalS10 promoter from M. rotundifolia 'Noble'; PVvCalS10, CalS10 promoter from V. vinifera 'Chardonnay'; and PN::GUS, nonpromoter vector pBI121. B, Histochemical assay for GUS expression. Grapevine leaves of $V$. vinifera 'Thompson seedless' were agro-infiltrated with each of the plasmids. GUS staining was performed after $24 \mathrm{~h}$ of inoculation with water or P. viticola. Water, control treatment; PV, infected by P. viticola. C, Quantitative analysis of GUS expression. Mean GUS activity \pm standard deviation $( \pm$ S.D. $)$ is averaged from three independent experiments $(n=3)$. S.D. is indicated on each bar. * indicates a significant difference $(P=0.05)$ of GUS activity. 
muscadine grapes. However, the cis-acting element involved in heat stress responsiveness, the $c i s$-acting element involved in lowtemperature responsiveness, and MYB binding site were not found in the muscadine grapes and the number of GARE-motifs was less in the muscadine grapes than in other cultivars (Table 2).

Analyzing the promoter sequences of CalS1O revealed that a small fragment deletion, three small fragment insertions, and more than 50 SNPs were found in DM-immune 'Noble' and 'Carlos', when they were compared with those in other cultivars. Although, hormone-regulated elements, such as the abscisic acid-responsive element ABRE, the MeJA-responsive element CGTCA-motif, the gibberellin-responsive element GARE-motif, and the salicylic acidresponsive element TCA, also existed in the CalS10 promoters, the GARE-motif could only be found in non-muscadine grapes. In addition, the ethylene-responsive element ERE and fungal elicitor responsive element Box-W1 were found in the CalS10 promoter region, while it did not appear in CalS1 promoters (Table 3).

Regulatory analysis of PCalS1 and PCalS10 after inoculation by $P$. viticola. To further study the pathogen regulation pattern of CalS1 and CalS10, the promoters which have been amplified from the DM-immune $M$. rotundifolia 'Noble' and the susceptible $V$. vinifera 'Chardonnay' were subcloned into a binary expression vector with $\beta$-glucuronidase (GUS) coding region (Fig. 4A) and transiently transformed into $V$. vinifera 'Thompson Seedless' leaves, respectively. Histochemical staining assay revealed that the GUS activity could be significantly observed in $35 \mathrm{~S}$ positive control, while it was varied among different combinations of CalS promoters. The expression of the GUS in PMrCalS1 and PMrCalS10 was induced by $P$. viticola inoculation, while no significant change was observed in $P V v C a l S 1$ or $P V v C a l S 10$ under the same treatment (Fig. 4B). The quantification analysis of GUS activity also clearly demonstrated that they were significantly up-regulated by PMrCalS1 and PMrCalS1O after P. viticola infection. However, there were no significant difference in GUS activities between water treatment and $P$. viticola inoculation in grapevine leaves with PN (nonpromoter), 35S, PVvCalS1, and PVvCalS10 (Fig. 4C).

Regulation of CalS1 and CalS10 by ABA and GA. To further study the role of CalS1 and CalS10 in responding to ABA treatment, the immune $M$. rotundifolia 'Noble' and the susceptible $V$. vinifera 'Chardonnay' were treated with $100 \mu \mathrm{M}$ ABA. Twelve hours after ABA treatment, the expression of CalS1 was significantly up-regulated (sixfold) in 'Noble', but significantly down-regulated in 'Chardonnay'. However, the expression of CalS10 was not affected by the ABA treatment in either cultivar (Fig. 5A). The histochemical staining assay and quantification analysis also showed that the expression of the PMrCalS1-GUS was increased, but the expression of the PVvCalS1-GUS was decreased after ABA treatment. No significant GUS changes were observed under the CalS10 promoter after ABA treatment (Fig. 5B and C).

In terms of the GA treatment assay, the expression of $P V v$ CalS10$G U S$ was induced, but the expression of the PVvCalS1-GUS was suppressed. No significant GUS changes were observed under the PMrCalS1 and the PMrCalS10 promoter (Fig. 5B and C).
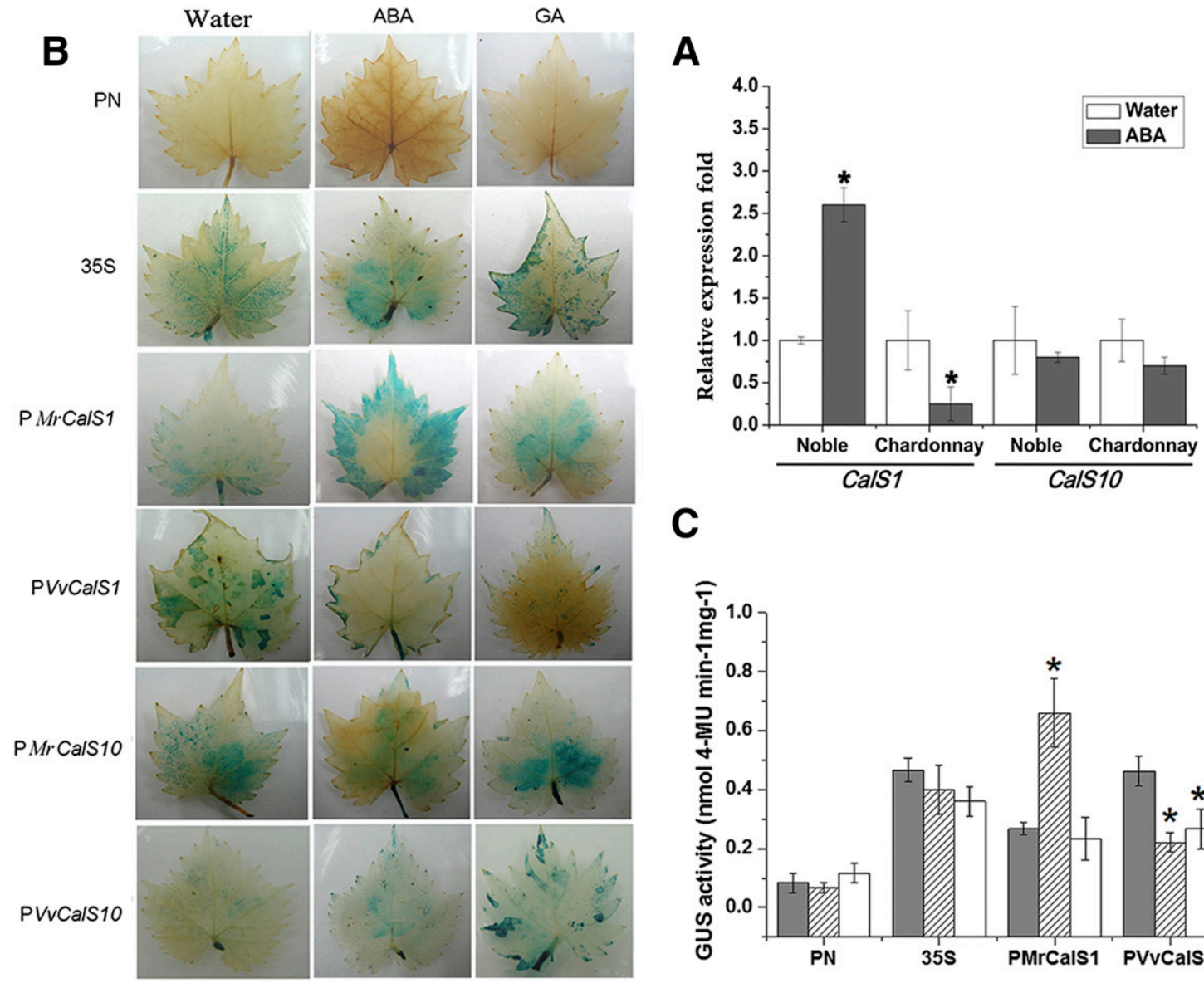

C

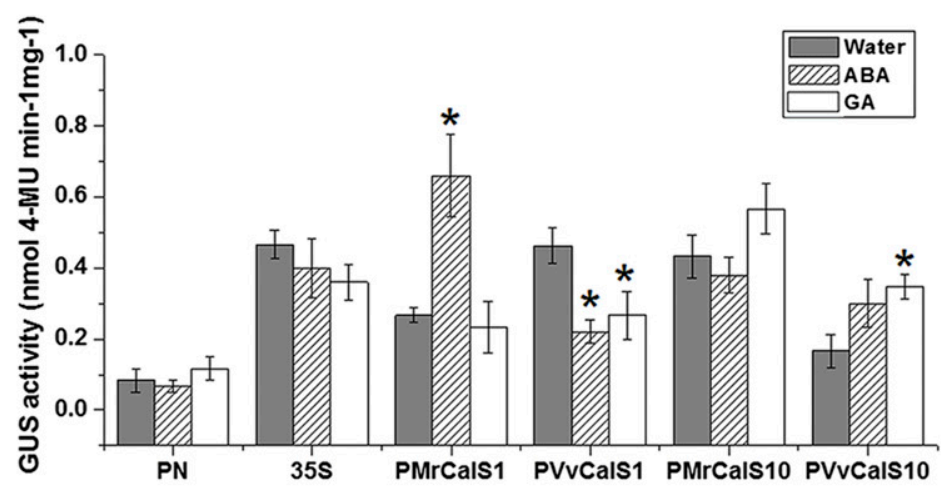

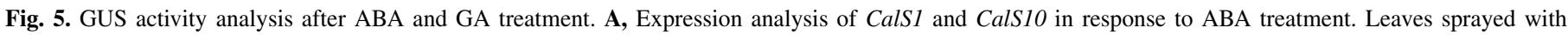

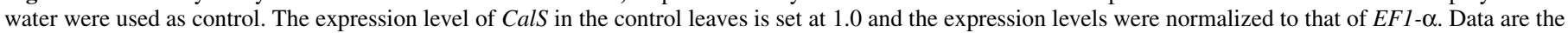

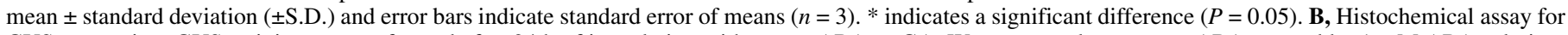

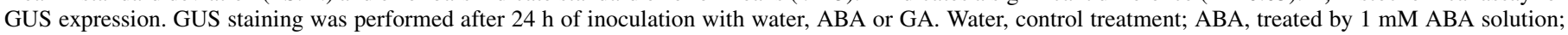

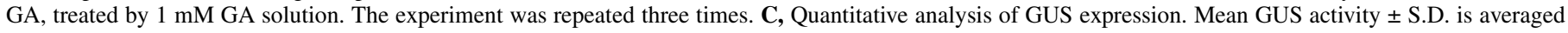
from three independent experiments $(n=3)$. S.D. is indicated on each bar. * indicates a significant difference $(P=0.05)$ of GUS activity. 


\section{DISCUSSION}

By comparing the sequence of A. thaliana callose synthase genes, eight $V v C a l S$ genes were identified in the $V$. vinifera 'Pinot Noir' genome. All grapevine callose synthases appear to be membrane proteins, with 7 to 10 transmembrane domains. Among the eight $\mathrm{CalS}$ genes tested in grapevine leaves, CalS5 was expressed at a very low level. It is possible that CalS5 plays a role in other tissues, as it has been reported that the expression of AtCalS genes was regulated in a tissue-specific manner (Dong et al. 2008).

In A. thaliana, AtCalS 1, 5, 9, 10, and 12 participate in defense response to pathogen infection (Dong et al. 2008). AtCalS3, 7, and 11 play key roles in plant growth and reproduction (Enns et al. 2005; Vatén et al. 2011; Xie et al. 2011). In this study, grapevine CalS1, 3, $7,8,9,10$, and 11 were all up-regulated by $P$. viticola in all the cultivars tested, suggesting that these $C a l S$ genes might be involved in defense responses against $P$. viticola attack.

It has been reported that callose deposition could be detected in the stomata (in the guard cell) around the germinating zoospores within $24 \mathrm{~h}$ after inoculation (Gindro et al. 2003), and the quantity of callose deposition was correlated to the resistance levels of grapevines (Yu et al. 2012). For example, the DM-immune $M$. rotundifolia showed stronger callose deposition than less resistant grape species $24 \mathrm{~h}$ after $P$. viticola inoculation. Gene expression analysis indicated that CalS1 and CalS10 were raised quickly in $M$. rotundifolia 'Carlos' and 'Noble' grapevines. CalS3 may also play a role in the early callose deposition as its expression increased significantly in response to pathogen infection. In the previous study, no callose deposits were observed in the DMsusceptible V. vinifera grapevines (Yu et al. 2012). However, gene expression analysis showed a significant up-regulation of $\mathrm{CalS} 3$ and 8 in susceptible $V$. vinifera 'Chardonnay' as early as 6 hpi, even though they did not lead to the callose deposition in the DMsusceptible grapevine.

Previous studies showed that the callose deposition could also be observed around the necrosis of cells surrounding and pathogen haustoria 24 hpi (Gindro et al. 2003; Yu et al. 2012). CalS1 and CalS10 were continuously induced after 24 hpi while significant down-regulations were detected in CalS7, 8,9, and 11. It is clear that the pathogen regulation of callose synthase genes in grapevine was complicated, which is consistent with other reports that callose synthesis played multiple roles in response to pathogen attack (Dong et al. 2008; Jacobs et al. 2003). Additionally, multiple callose synthase genes were up-regulated at the same time point in the same cultivar, suggesting a complementary function of these CalS proteins.

Callose deposition was also reported to be tightly correlated with the plant hormone abscisic acid (ABA) (Ton and Mauch-Mani 2004; Ton et al. 2005). A primed accumulation of callose was shown to be regulated by an ABA-dependent signaling pathway (Ton and Mauch-Mani 2004). Flors et al. (2008) found that the low ABA content in the wild-type A. thaliana led to a weak induction of callose deposition. Our results were consistent with those studies and suggested a possible connection between the expression of selected callose synthase genes and ABA level in grapevine. It has been reported that $\beta$-aminobutyric acid (BABA) could induce resistance against oomycetes in lettuce and $A$. thaliana (Pajot et al. 2001; Zimmerli et al. 2000), as well as in grapevine 'Chasselas' (Hamiduzzaman et al. 2005). This enhanced resistance in grapevine against $P$. viticola was associated with high callose deposition. In our study, a correlation between ABA treatment and CalS1 expression was observed in the DM-immune muscadine grapes. A dual role of another plant growth regulator GA was reported in A. thaliana. The exogenous application of GA resulted in enhanced resistance to Pseudomonas syringae pv. tomato DC3000 and susceptibility to A. brassicicola in A. thaliana (Bari R and Jones 2009). Our results suggested that both grapevine CalS1 and CalS10 were regulated by GA. To elucidate the regulation mechanism of
CalS, further studies are necessary to better understand the relationship between CalS expression and the hormone signals during $P$. viticola infection.

\section{ACKNOWLEDGMENTS}

This work was supported by the China Agricultural University Scientific Fund (Grant No. 2012RC019), China Agriculture Research System (CARS30-yz-2), and National Natural Science Foundation of China (Grant No. $31471754)$.

\section{LITERATURE CITED}

Bari, R., and Jones, J. D. G. 2009. Role of plant hormones in plant defense responses. Plant Mol. Biol. 69:473-488.

Blümke, A., Falter, C., Herrfurth, C., Sode, B., Bode, R., Schafer, W., Feussner, I., and Voigt, C. A. 2014. Secreted fungal effector lipase releases free fatty acids to inhibit innate immunity-related callose formation during wheat head infection. Plant Physiol. 165:346-358.

Boso, S., and Kassemeyer, H. H. 2008. Different susceptibility of European grapevine cultivars for downy mildew. Vitis 47:39-49.

Brown, I., Trethowan, J., Kerry, M., Mansfield, J., and Bolwell, G. P. 1998. Localization of components of the oxidative cross-linking of glycoproteins and of callose synthesis in papillae formed during the interaction between non-pathogenic strains of Xanthomonas campestris and French bean mesophyll cells. Plant J. 15:333-343.

Brownfield, L., Ford, K., Doblin, M. S., Newbigin, E., Read, S., and Bacic, A. 2007. Proteomic and biochemical evidence links the callose synthase in Nicotiana alata pollen tubes to the product of the NaGSL1 gene. Plant J. 52: 147-156.

Chen, X. Y., and Kim, J. Y. 2009. Callose synthesis in higher plants. Plant Signal. Behav. 4:489-492.

Clay, N. K., Adio, A. M., Denoux, C., Jander, G., and Ausubel, F. M. 2009. Glucosinolate metabolites required for an Arabidopsis innate immune response. Science 323:95-101.

Doblin, M. S., De Melis, L., Newbigin, E., Bacic, A., and Read, S. M. 2001. Pollen tubes of Nicotiana alata express two genes from different $\beta$-glucan synthase families. Plant Physiol. 125:2040-2052.

Dong, X., Hong, Z., Chatterjee, J., Kim, S., and Verma, D. P. 2008. Expression of callose synthase genes and its connection with Nprl signaling pathway during pathogen infection. Planta 229:87-98.

Dong, X., Hong, Z., Sivaramakrishnan, M., Mahfouz, M., and Verma, D. P. S. 2005. Callose synthase (CalS5) is required for exine formation during microgametogenesis and for pollen viability in Arabidopsis. Plant J. 42: 315-328.

Donofrio, N. M., and Delaney, T. P. 2001. Abnormal callose response phenotype and hypersusceptibility to Peronospora parasitica in defensecompromised Arabidopsis nim1-1 and salicylate hydroxylase-expressing plants. Mol. Plant-Microbe Interact. 14:439-450.

Ellinger, D., Naumann, M., Falter, C., Zwicowics, C., Jamrow, T., Manisseri, C., Somerville, S. C., and Voigt, C. A. 2013. Elevated early callose deposition results in complete penetration resistance to powdery mildew in Arabidopsis. Plant Physiol. 161:1433-1444.

Enns, L. C., Kanaoka, M. M., Torii, K. U., Comai, L., Okada, K., and Cleland, R. E. 2005. Two callose synthases, GSL1 and GSL5, play an essential and redundant role in plant and pollen development and fertility. Plant Mol. Biol. 58:333-349.

Enrique, R., Siciliano, F., Favaro, M. A., Gerhardt, N., Roeschlin, R., Rigano, L., Sendin, L., Castagnanaro, A., Vojnov, A., and Marano, M. R. 2011. Novel demonstration of RNAi in citrus reveals importance of citrus callose synthase in defence against Xanthomonas citri subsp. citri. Plant Biotechnol. J. 9:394-407.

Eshraghi, L., Anderson, J. P., Aryamanesh, N., Mccomb, J. A., Shearer, B. L., and Hardy, G. E. S. J. 2014. Defence signalling pathways involved in plant resistance and phosphite-mediated control of Phytophthora cinnamomi. Plant Mol. Biol. Rep. 32:342-356.

Flors, V., Ton, J., Van Doorn, R., Jakab, G., Garcia-Agustin, P., and Mauch-Mani, B. 2008. Interplay between JA, SA and ABA signalling during basal and induced resistance against Pseudomonas syringae and Alternaria brassicicola. Plant J. 54:81-92.

Gindro, K., Pezet, R., and Viret, O. 2003. Histological study of the responses of two Vitis vinifera cultivars (resistant and susceptible) to Plasmopara viticola infections. Plant Physiol. Biochem. 41:846-853.

Gomez-Gomez, L., Felix, G., and Boller, T. 1999. A single locus determines sensitivity to bacterial flagellin in Arabidopsis thaliana. Plant J. 18:277-284.

Guan, X., Zhao, H., Xu, Y., and Wang, Y. 2011. Transient expression of glyoxal oxidase from the Chinese wild grape Vitis pseudoreticulata can 
suppress powdery mildew in a susceptible genotype. Protoplasma 248: 415-423.

Hamiduzzaman, M. M., Jakab, G., Barnavon, L., Neuhaus, J. M., and Mauch-Mani, B. 2005. $\beta$-Aminobutyric acid-induced resistance against downy mildew in grapevine acts through the potentiation of callose formation and jasmonic acid signaling. Mol. Plant-Microbe Interact. 18: 819-829.

Henanff, G. L., Heitz, T., Mestre, P., Mutterer, J., Walter, B., and Chong, J. 2009. Characterization of Vitis vinifera NPR1 homologs involved in the regulation of pathogenesis-related gene expression. BMC Plant Biol. 9: 54-67.

Jacobs, A. K., Lipka, V., Burton, R. A., Panstuga, R., Strizhov, N., Schulze-Lefert, P., and Fincher, G. B. 2003. An Arabidopsis callose synthase, GSL5, is required for wound and papillary callose formation. Plant Cell 15:2503-2513.

Jefferson, R. A. 1987. Assaying chimeric genes in plants: The GUS gene fusion system. Plant Mol. Biol. Rep. 5:387-405.

Jiao, L., Zhang, Y., Wu, J., Zhang, H., and Lu, J. 2015. A novel U-box protein gene from "Zuoshanyi" grapevine (Vitis amurensis Rupr. cv.) involved in cold responsive gene expression in Arabidopsis thaliana. Plant Mol. Biol. Rep. 33:557-568.

Kelly, R., Register, E., and Hsu, M. J. 1996. Isolation of a gene involved in 1,3$\beta$-glucan synthesis in Aspergillus nidulans and purification of the corresponding protein. J. Bact. 178:4381-4391.

Lee, S. C., and Hwang, B. K. 2006. Identification and deletion analysis of the promoter of the pepper SAR8.2 gene activated by bacterial infection and abiotic stresses. Planta 224:255-267.

Li, H., Ge, X., Han, S., Sivasithamparam, K., and Barbetti, M. J. 2010. Histological responses of host and non-host plants to Hyaloperonospora parasitica. Eur. J. Plant Pathol. 129:221-232.

Li, Y. F., Zhu, R., and Xu, P. 2005. Activation of the gene promoter of barley1,3-glucanase isoenzyme GIII is salicylic acid (SA)-dependent in transgenic rice plants. J. Plant Res. 118:215-221.

Liu, J.-J., Ekramoddoullah, A. K. M., Piggott, N., and Zamani, A. 2005. Molecular cloning of a pathogen/wound-inducible PR10 promoter from Pinus monticola and characterization in transgenic Arabidopsis plants. Planta 221:159-169.

Livak, K. J., and Schmittgen, T. D. 2001. Analysis of relative gene expression data using real-time quantitative PCR and the $2^{-\triangle \Delta C T}$ method. Methods 25 : 402-408.

Luna, E., Pastor, V., Robert, J., Flors, V., Mauch-Mani, B., and Ton, J. 2011. Callose deposition: A multifaceted plant defense response. Mol. PlantMicrobe Interact. 24:183-193.

Malnoy, M., Venisse, J. S., Reynoird, J. P., and Chevreau, E. 2003. Activation of three pathogen-inducible promoters of tobacco in transgenic pear (Pyrus communis L.) after abiotic and biotic elicitation. Planta 216:802-814.

Murray, M. G., and Thompson, W. F. 1980. Rapid isolation of high molecular weight plant DNA. Nucleic Acids Res. 8:4321-4325.

Nishimura, M. T., Stein, M., Hou, B., Vogel, J. P., Edwards, H., and Somerville, S. C. 2003. Loss of a callose synthase results in salicylic aciddependent disease resistance. Science 301:969-972.
Pajot, E., Le Corre, D., and Silue, D. 2001. Phytograd and DL-beta-amino butyric acid (BABA) induce resistance to downy mildew (Bremia lactucae) in lettuce (Lactuca sativa L). Eur. J. Plant Pathol. 107:861-869.

Richmond, T. A., and Somerville, C. R. 2000. The cellulose synthase superfamily. Plant Physiol. 124:495-498.

Santos-Rosa, M., Poutaraud, A., Merdinoglu, D., and Mestre, P. 2008. Development of a transient expression system in grapevine via agroinfiltration. Plant Cell Rep. 27:1053-1063.

Tamura, K., Dudley, J., Nei, M., and Kumar, S. 2007. MEGA4: Molecular evolutionary genetics analysis (MEGA) software version 4.0. Mol. Biol. Evol. 24:1596-1599.

Thompson, J. D., Higgins, D. G., and Gibson, T. J. 1994. CLUSTAL W: Improving the sensitivity of progressive multiple sequence alignment through sequence weighting, positions-specific gap penalties and weight matrix choice. Nucleic Acids Res. 22:4673-4680.

Ton, J., Flors, V., and Mauch-Mani, B. 2009. The multifaceted role of ABA in disease resistance. Trends Plant Sci. 14:310-317.

Ton, J., Jakab, G., Toquin, V., Flors, V., Lavicoli, A., Maeder, M. N., Metraux, J.-P., and Mauch-Mani, B. 2005. Dissecting the $\beta$-aminobutyric acid induced priming pathways in Arabidopsis. Plant Cell 17:987-999.

Ton, J., and Mauch-Mani, B. 2004. Beta-amino-butyric acid-induced resistance against necrotrophic pathogens is based on ABA-dependent priming for callose. Plant J. 38:119-130.

Vatén, A., Dettmer, J., Wu, S., Stierhof, Y. D., Miyashima, S., Yadav, S. R., Roberts, C. J., Campilho, A., Bulone, V., Lichtenberger, R., Lehesranta, S., Mahonen, A. P., Kim, J. Y., Jokitalo, E., Sauer, N., Scheres, B., Nakajima, K., Carlsbecker, A., Gallagher, K. L., and Helariutta, Y. 2011. Callose biosynthesis regulates symplastic trafficking during root development. Dev. Cell 21:1144-1155.

Verma, D. P. S., and Hong, Z. 2001. Plant callose synthase complexes. Plant Mol. Biol. 47:693-701.

Xie, B., Wang, X., Zhu, M., Zhang, Z., and Hong, Z. 2011. CalS7 encodes a callose synthase responsible for callose deposition in the phloem. Plant J. 65:1-14.

Xu, W., Yu, Y., Ding, J., Hua, Z., and Wang, Y. 2010. Characterization of a novel stilbene synthase promoter involved in pathogen- and stress-inducible expression from Chinese wild Vitis pseudoreticulata. Planta 231:475-487.

Yamaguchi, T., Hayashi, T., Nakayama, K., and Koike, S. 2006. Expression analysis of genes for callose synthases and Rho-type small GTP-binding proteins that are related to callose synthesis in rice anther. Biosci. Biotechnol. Biochem. 70:639-645.

Yang, Y., Ling, X., Chen, T., Cai, L., Liu, T., Wang, J., Fan, X., Ren, Y., Yuan, H., Zhu, W., Zhang, B., and Ma, D. P. 2014. A cotton Gbvdr5 gene encoding a leucine-rich-repeat receptor-like protein confers resistance to Verticillium dahliae in transgenic Arabidopsis and upland cotton. Plant Mol. Biol. Rep. 2014:1-15.

Yu, Y., Zhang, Y., Yin, L., and Lu, J. 2012. The mode of host resistance to Plasmopara viticola infection of grapevines. Phytopathology 102:1094-1101.

Zimmerli, L., Jakab, G., Metraux, J. P., and Mauch-Mani, B. 2000. Potentiation of pathogen-specific defence mechanisms in Arabidopsis by betaaminobutyric acid. Proc. Natl. Acad. Sci. USA 97:12920-12925. 